\title{
Emerging understanding of apoptosis in mediating mesenchymal stem cell therapy
}

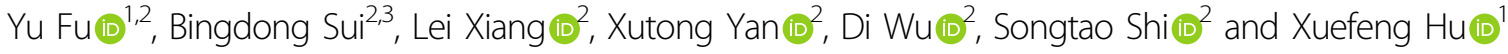

\begin{abstract}
Mesenchymal stem cell transplantation (MSCT) has been recognized as a potent and promising approach to achieve immunomodulation and tissue regeneration, but the mechanisms of how MSCs exert therapeutic effects remain to be elucidated. Increasing evidence suggests that transplanted MSCs only briefly remain viable in recipients, after which they undergo apoptosis in the host circulation or in engrafted tissues. Intriguingly, apoptosis of infused MSCs has been revealed to be indispensable for their therapeutic efficacy, while recipient cells can also develop apoptosis as a beneficial response in restoring systemic and local tissue homeostasis. It is notable that apoptotic cells produce apoptotic extracellular vesicles (apoEVs), traditionally known as apoptotic bodies (apoBDs), which possess characterized miRnomes and proteomes that contribute to their specialized function and to intercellular communication. Importantly, it has been demonstrated that the impact of apoEVs is long-lasting in health and disease contexts, and they critically mediate the efficacy of MSCT. In this review, we summarize the emerging understanding of apoptosis in mediating MSCT, highlighting the potential of apoEVs as cell-free therapeutics.
\end{abstract}

\section{Facts}

- In the human body, 50-70 billion cells die every day, during which plenty of apoptotic extracellular vesicles (apoEVs) are produced and are involved in tissue homeostasis maintenance and disease development.

- Infused mesenchymal stem cells (MSCs) disappear soon in the recipients, which are further revealed to undergo extensive apoptosis.

- Transplanted apoptotic MSCs and apoEVs interact with recipient cells, which improve tissue regeneration and immunomodulation.

Correspondence: Songtao Shi (shisongtao@mail.sysu.edu.cn) or

Xuefeng Hu (bioxfh@fjnu.edu.cn)

${ }^{1}$ Fujian Key Laboratory of Developmental and Neural Biology \& Southern

Center for Biomedical Research, College of Life Sciences, Fujian Normal

University, Fuzhou, Fujian 350117, China

${ }^{2}$ South China Center of Craniofacial Stem Cell Research, Guanghua School and

Hospital of Stomatology, Sun Yat-sen University, Guangzhou, Guangdong

510055, China

Full list of author information is available at the end of the article

These authors contributed equally: Yu Fu, Bingdong Sui

Edited by $Y$. Shi

\section{Open questions}

- Do endogenous MSCs autonomously execute apoptosis and result in abundant apoEV production that contribute to systemic and local tissue homeostasis maintenance?

- Whether infused MSCs undergo other cell death processes attributed to diverse physiological and pathological contexts, such as the autophagic cell death, the necroptosis and the pyroptosis?

- Whether EVs produced by multiple cell death processes have differential regulatory and therapeutic effects?

\section{Introduction}

Since mesenchymal stem cells (MSCs) were originally discovered in the early 1970s, these primitive cells have been known to give rise to multilineage descendants while retaining the capacity to self-renew ${ }^{1-3}$. In recent years, increasing understanding of these cells as crucial contributors to organogenesis and immunomodulation has led to the development and application of preclinical and

\section{(c) The Author(s) 2021}

(c) Open Access This article is licensed under a Creative Commons Attribution 4.0 International License, which permits use, sharing, adaptation, distribution and reproduction c. in any medium or format, as long as you give appropriate credit to the original author(s) and the source, provide a link to the Creative Commons license, and indicate if changes were made. The images or other third party material in this article are included in the article's Creative Commons license, unless indicated otherwise in a credit line to the material. If material is not included in the article's Creative Commons license and your intended use is not permitted by statutory regulation or exceeds the permitted use, you will need to obtain permission directly from the copyright holder. To view a copy of this license, visit http://creativecommons.org/licenses/by/4.0/. 
clinical studies based on MSC transplantation (MSCT) ${ }^{4-9}$. While the therapeutic effects of MSCT on degenerated organs/tissues and immune disorders have been studied in some detail, the mechanisms by which transplanted MSCs interplay with recipients to provoke therapeutic cascades after administration remain less defined, resulting in bottleneck problems on the path toward controllable and precise therapies. Along with various therapeutic cytokines, MSCs release multiple extracellular vesicles (EVs), which are membrane-bound structures of endosomal origin or shed from the plasma membrane $\mathrm{e}^{10,11}$. As carriers of bioactive molecules and organelles transferred to recipient cells, EVs possess specific biological functions and have immense effects in MSCT $^{10,12}$. Accumulating studies have reported that MSC-derived EVs exert beneficial effects in various disease models through transferring proteins and microRNAs (miRNAs), constituting one paracrine mechanism of MSCT $^{13-16}$.

Apoptosis, a physiological and autonomous clearance process used by an organism to remove unwanted cells, was also first described in the early 1970s and subsequently found to play significant roles in development, tissue homeostasis, aging, and pathogenesis ${ }^{17-20}$. During the execution of apoptosis, apoptotic EVs (apoEVs), originally known as apoptotic bodies (apoBDs), are formed by membrane blebbing or protrusion with specific intracellular content distribution, and have emerged as regulators of multiple biological processes rather than mere debris $^{21}$. In particular, apoEVs have been shown to critically regulate $\mathrm{T}$-cell and macrophage immune function, as well as promote tissue recovery including skin regeneration and vascular protection ${ }^{2-26}$. Notably, increasing evidence has suggested that transplanted MSCs undergo extensive apoptosis, during which the released apoEVs serve as indispensable therapeutic mediators ${ }^{27-30}$. Functioning through engulfment or dynamically interacting with recipient cells, apoEVs exert regulatory effects based on a fine-tuned molecular network ${ }^{24,30}$. Importantly, direct delivery of apoptotic MSCs or apoEVs produced by allogeneic apoptotic MSCs has further been revealed to possess advantages over viable $\mathrm{MSCs}^{31,32}$. Accordingly, MSC-derived apoEV transplantation holds the promise of counteracting various diseases including myocardial infarction (MI), osteoporosis, graft-versus-host disease (GvHD), colitis, and more ${ }^{22,30-34}$. Here we review the cutting-edge knowledge regarding apoptosis and apoEVs in mediating MSC therapy.

\section{Historical perspectives on MSCs and MSCT}

MSCs are non-hematopoietic stromal cells which were originally isolated and identified in postnatal mammalian bone marrow (BMMSCs) by Friedenstein et al. ${ }^{2}$. They possess plastic adherence and clonogenic properties with multilineage differentiation capabilities in vitro ${ }^{3}$. Enlightened by BMMSC discoveries, a series of MSCs were isolated and identified from a variety of mammalian tissues including the adipose tissue, umbilical cord, tendons, and the orofacial region ${ }^{35-41}$. These MSCs from other sources not only display features typical of BMMSCs but also exhibit the functional characteristics associated with their tissue-specific origins and locations ${ }^{42-46}$. In addition to their self-renewal and differentiation potential, MSCs are further characterized by potent immunomodulatory properties. For example, they suppress proliferation and activation of immune cells, particularly $\mathrm{T}$ cells $\mathrm{s}^{9,47-52}$. Several classical markers have been generally used to identify human BMMSCs by their surface antigens, including but not limited to CD105, CD146, CD271, and STRO-1, while CD11b, CD31, CD34, and CD45 serve as negative markers ${ }^{53-56}$. Other tissue-specific MSCs, such as dental pulp stem cells (DPSCs), are derived from neural crest cells in early head development and express neurovascular-associated markers including neuron glia 2 (NG2) and alpha-smooth muscle actin ( $\alpha$-SMA), as they contribute to neurogenesis and angiogenesis ${ }^{57,58}$. Furthermore, functional markers for certain MSC subpopulations, such as nestin, Gli1, leptin receptor (LepR), and programmed cell death 1 (PD1), have been revealed to control MSC proliferation and differentiation in vivo ${ }^{57,59-61}$.

In light of their self-renewal, multilineage differentiation, and immunoregulatory properties, MSCs have been widely used as cellular therapeutics in tissue regeneration and treatment of immune disorders, which has prompted a spectrum of clinical studies. The first clinical therapeutic application of allogenic MSCs dates back to the early 2000s with Horwitz's study in which six children with severe osteogenesis imperfecta received transplantation of allogeneic BMMSCs ${ }^{4}$. The results demonstrated therapeutic effects including acceleration of the tissue growth during the first 6 months post-infusion ${ }^{4}$. Subsequently, Le Blanc et al. have accomplished the first clinical trial showing significant efficacy of MSCs in treating human $\mathrm{GvHD}^{5}$. To date, many clinical trials aimed at tissue regeneration have been initiated or accomplished, applying MSCT to treat ischemic heart failure, osteonecrosis, osteoarthritis, and more ${ }^{62-64}$. Moreover, in situ transplantation of DPSCs has been applied for dental pulp regeneration in humans, the success of which may be attributed to their capacity to give rise to neurovascular tissue as noted above ${ }^{65}$. In parallel, extensive clinical trials for treatment of immune disorders have been conducted, such as in GvHD, systemic lupus erythematosus (SLE), and multiple sclerosis ${ }^{6-68}$. To date, more than 400 studies on MSC immunomodulation have been registered in clinical trial databases. Collectively, these trials establish that the recognition of MSC-specific characteristics represents an important basis for future clinical translational medicine. 


\section{Therapeutic mechanisms of MSC transplantation}

While MSCT has exhibited extensive biological effects that promote regenerative repair and immunoregulation, the cellular and molecular mechanisms underlying the potential therapeutic interplay between transplanted MSCs and recipient components remain elusive. Through direct effects based on engraftment and differentiation, as well as indirect effects based on paracrine mechanisms including cytokines and EVs, MSCs exert immense therapeutic efficacy $^{13-16}$ (Fig. 1). It has been widely reported in MI that intravenous-infused MSCs engraft and differentiate into cardiomyocytes while recruiting endogenous cardiac stem cells, which attenuate the progressive deterioration of the heart and improve cardiac function ${ }^{6,7,69}$. In parallel, transplanted MSCs have dynamic interactions with the local stem cell niche, which also contributes to the tissue recovery ${ }^{70}$. While the above findings have demonstrated that MSCT repairs tissue injury in various diseases via regulating the engrafted tissue, answers to the questions of how MSCs engraft in recipient tissues and how long they remain in these recipient tissues remain elusive. It has been proposed that homing of transplanted MSCs relies on recruitment by endogenous cells ${ }^{71}$, but there has been no direct evidence to prove that the engrafted MSCs are still alive to exert effects (Fig. 1).
Intriguingly, further studies have reported that transplanted MSCs are trapped in the lung and become undetectable within $24 \mathrm{~h}$ post-injection, which subsequently undergoing extensive apoptosis over the short term $^{6,28,30}$ (Fig. 1). Accordingly, revealing the bona fide mechanisms of MSCT will be highly significant for improving strategy for tissue regeneration and homeostatic maintenance.

With regard to paracrine mechanisms, pioneering studies by Gnecchi et al. have shown that injection of conditioned medium (CM) of MSCs remarkably improves cardiac performance ${ }^{72,73}$. Subsequently, increasing evidence has suggested that infused MSCs secrete a serious of cytokines, including TNF-stimulated gene 6 protein (TSG-6), prostaglandin E2 (PGE2), insulin-like growth factor 2 (IGF-2), indoleamine 2,3-dioxygenase (IDO) metabolite kynurenine, vascular endothelial growth factor (VEGF), and basic fibroblast growth factor (bFGF), among others, to regulate recipient cells in immunomodulation, angiogenesis, and migration ${ }^{6,28,74-79}$. Furthermore, as MSCs secrete a large amount of EVs, including exosomes (with diameters in the range of $30-100 \mathrm{~nm}$ ) and microvesicles (diameters within the range of 50-1000 nm), EV release has been increasingly recognized as a critical mechanism for the transfer of bioactive molecules in MSC

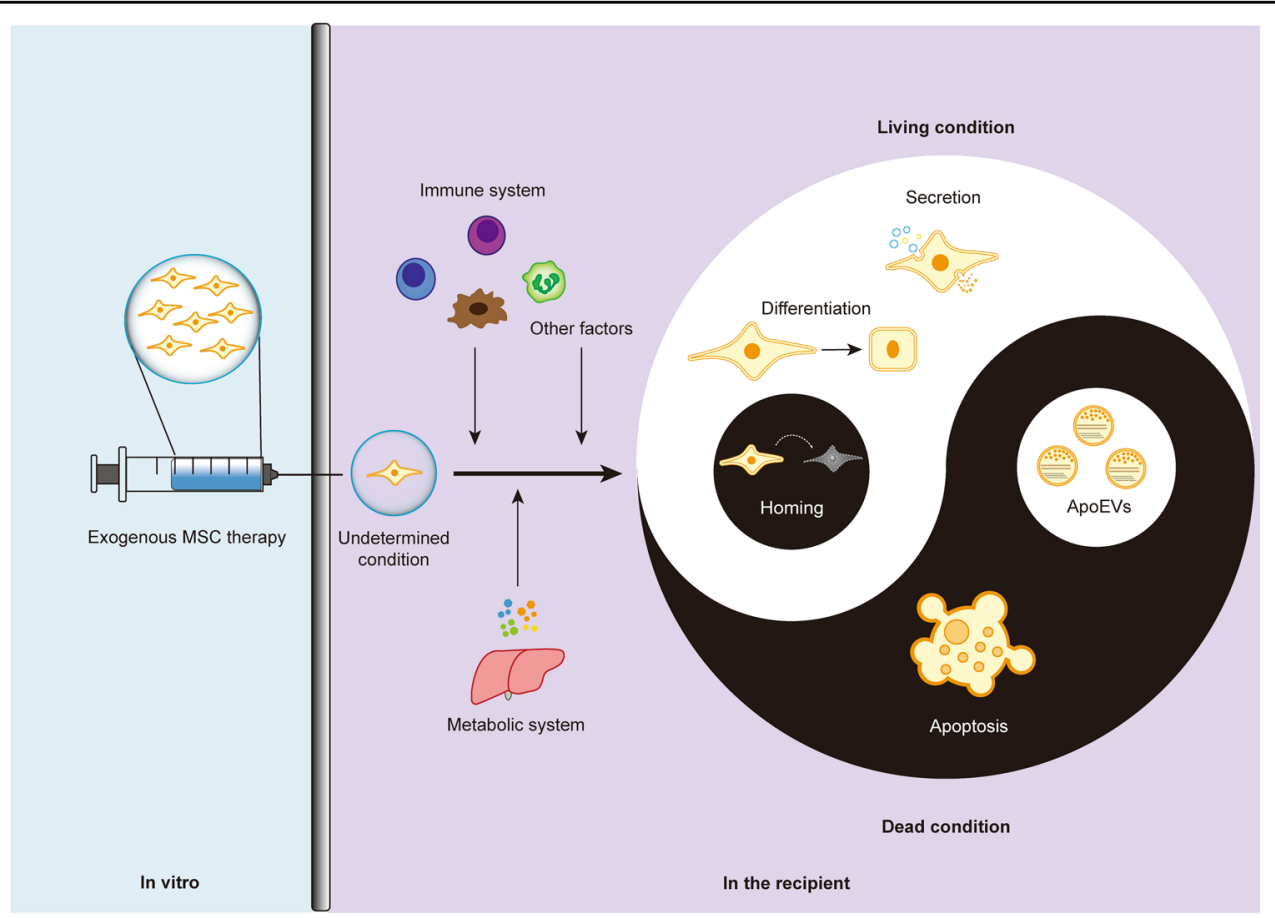

Fig. 1 Live-dead decision of MSCs in therapy. After infusion, exgenous MSCs in the undetermined condition confront with the stimuli of multiple factors inculding immunological, metabolic, and other cues. Subsequently, the MSCs can be alive to exert therapeutic effects based on differentiation and secretion, which also can undergo apoptosis to regulate immune responses. ApoEVs have been demonstrated to be a noval and potent therapeutic in translational medicine. The above condition of live-dead decision for infused MSCs based on current recognitions has emerged as a representative paradigm of Taichi. 
therapy ${ }^{10,21}$. It has been reported that infused MSCs, through exosomes transferring Fas protein, modulate the intra-/extracellular balance of miR-29b in recipient stem cells and recover DNA methyltransferase 1 (Dnmt1)mediated Notch promoter hypomethylation and Notch signaling activation, indicating epigenetic regulation of recipient stem cells by MSCT-mediated paracrine mechanisms ${ }^{80}$. It has further been documented that miR151-5p secreted within exosomes by donor MSCs can be transferred to endogenous MSCs in systemic sclerosis mice to inhibit interleukin 4 receptor $\alpha$ (IL4R $\alpha$ ) expression and block mammalian target of rapamycin (mTOR) pathway activation, which rescues endogenous MSC functional defects in treating osteoporosis ${ }^{81}$. MSCTmediated exosomal or microvesicle transfer of functional proteins and non-coding RNAs has been widely reported in treating many other diseases, including MI, acute lung injury, and experimental colitis ${ }^{13,15,82-84}$.

Considering all these studies, the bona fide regulatory mechanisms of MSCT have increasingly been revealed, which has further shaped our understanding of the behaviors of MSCs in translational medicine. While there is a lack of long-term engraftment after MSCT, there is also potent secretion of EVs. Whether and how these two processes are linked in transplanted MSCs remains an intriguing question. In this regard, recent studies on release EVs, particularly in the course of MSC apoptosis, have provided a new perspective on MSC therapy, as stated below.

\section{Apoptosis in organismal homeostasis and therapeutic processes of MSCT}

As the most prominent mode of programmed cell death (PCD), apoptosis has been recognized as a physiological process that is widely involved in development, tissue homeostasis, aging, and pathogenesis ${ }^{85-87}$. During apoptosis, a cell undergoes a serious of well-characterized morphological changes including cytoplasmic shrinkage, membrane blebbing or protrusion, and nuclear condensation ${ }^{17,20,85,87}$. Subsequently, it has been revealed that active caspases cleave Rho effector protein ROCK1, which generates a truncated kinase with biological activity for actin-myosin remodeling and cell contractility ${ }^{88}$. Then, the cellular membrane gradually protrudes accompanied by blebbing and is fragmented in the final formation of apoptotic debris and apoEVs ${ }^{21}$.

Apoptosis is closely correlated with the immune balance of an organism. Because immune systems would be overactivated if immunogenic intracellular materials were released, it is necessary to clear apoptotic cells or apoEVs quickly enough to prevent secondary necrosis and thereby remain tissue homeostasis ${ }^{19,89}$. Furthermore, apoptosis has proven to be critical for attenuation of autoimmune reactions, not only by directing phagocytic cells into an anti-inflammatory phenotype, but also by regulating adaptive immune responses mediated by $\mathrm{T}$ cells and $\mathrm{B}$ cells $^{22,90-93}$. Importantly, inefficient engulfment of endogenous apoptotic cells can cause a variety of autoimmune diseases, such as SLE, severe anemia, and chronic arthritis $^{94-98}$.

The delicate equilibrium between stem cell-mediated proliferation (i.e., compensatory proliferation) and the neighboring stem or somatic cell apoptosis plays an indispensable role in tissue regeneration after injury ${ }^{99}$. Studies have reported that WNT and c-Jun amino-terminal kinase (JNK) signaling induced by surrounding apoptotic stimuli contribute to compensatory proliferation $^{100,101}$. Recent evidence has also shown that apoptotic epithelial stem cells facilitate adjacent stem cell proliferation by caspase-dependent production of WNT8acontaining apoEVs, suggesting that the plasticity of stem cells enables them to adapt to tissue homeostatic and regenerative needs upon sensing apoptotic signaling ${ }^{102}$. Furthermore, deletion of the pro-apoptotic protein ARTS in intestinal stem cells enhances WNT signaling and stimulates augmented cell proliferation in the tissue, indicating dynamic regulation of tissue homeostasis by apoptotic signaling interactions within the stem cell niche $^{103}$. Apoptotic signaling has also been identified as crucial to maintaining hepatic and neural tissue regeneration $^{104-106}$. In addition to the contribution to cell proliferation, apoptotic cells can trigger non-autonomous apoptosis of surrounding cells via production of tumor necrosis factor (TNF) homolog Eiger to activate JNK pathway in Drosophila ${ }^{107}$, indicating the complex nature of the roles of apoptosis plays in tissue maintenance.

In MSCT, apoptosis is also an important biological process that has gradually been noticed (Fig. 1). It has been reported that infused human MSCs (hMSCs) are trapped and disappear in the lung, whereas the antiinflammatory protein TSG-6 is upregulated in the lung to prevent injury ${ }^{6}$. It has further been documented that infused MSCs decrease markedly in tissues with extensive apoptosis within $24 \mathrm{~h}$, which intriguingly promotes their secretion of TSG-6 to prevent hypertrophic scar formation $^{28}$. As shown by the evidence in vitro, MSCs activated complement system and suffered injury after serum contact, while are further proposed that infused MSCs are involved in interaction with the recognition and attack of complement in vivo ${ }^{108}$. Apoptosis of infused MSCs can also be induced by pro-inflammatory $\mathrm{T}$ cells via interferon-gamma (IFN- $\gamma$ ) and TNF- $\alpha^{27}$. Moreover, perforin-dependent apoptotic execution of transplanted MSCs has been demonstrated to be essential for the initiation of MSC-induced immunosuppression, which has been further confirmed in patients with GvHD: only those with high cytotoxic activity against MSCs respond to MSC infusion ${ }^{29}$. Importantly, release of apoEVs by 


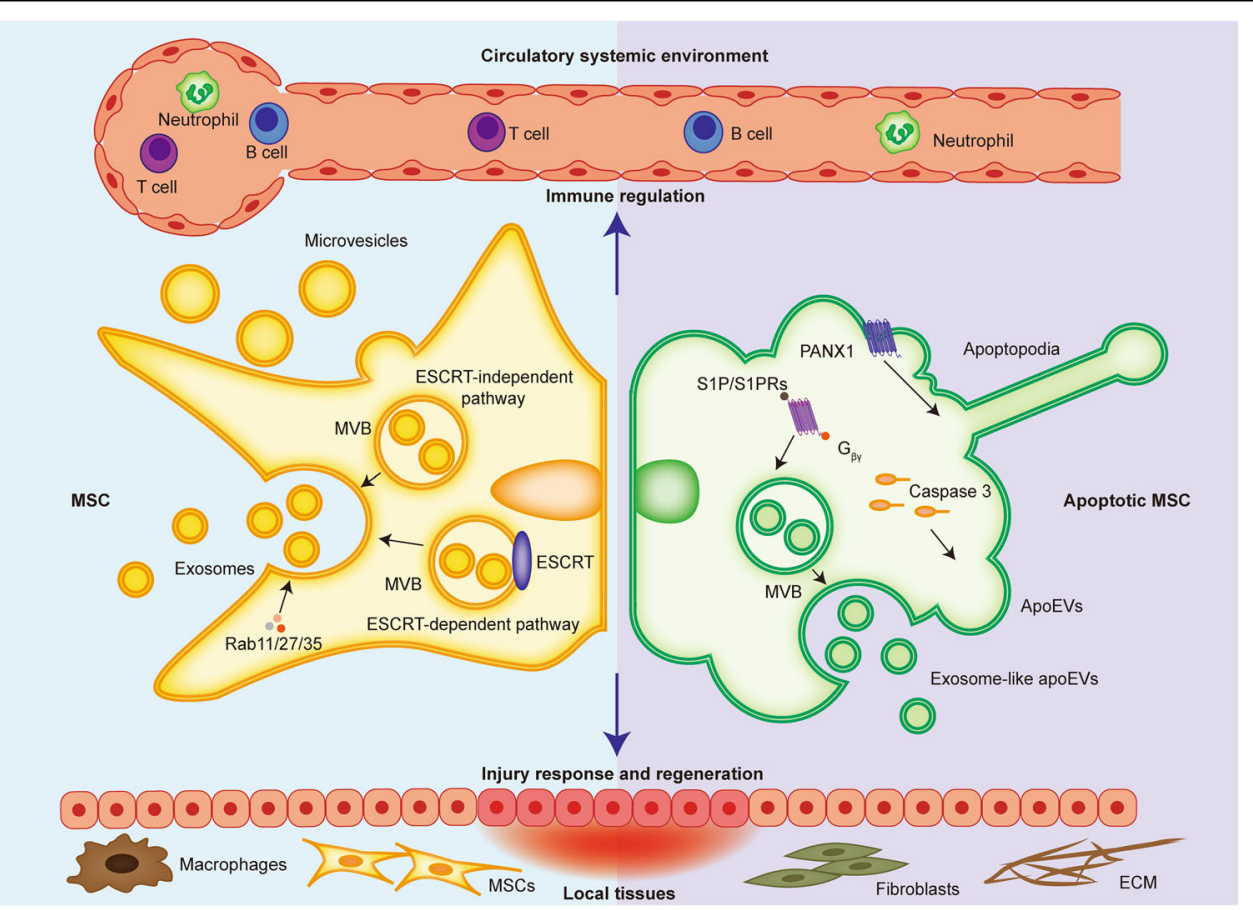

Fig. 2 Generation and functionality of EVs from normal and apoptotic cells. From normal cells, exosomes are formed through exocytosis of endosomal membrane based on ESCRT-dependent and -independent mechanisms, which subsequently secrete exosomes via Rab11/27/35. Microvesicles are shed from the plasma membrane. In contrast, apoEVs are released from apoptotic cells based on multiple mechanisms including (S1P)/S1PRs- and Caspase 3-depended apoEV release as well as PANX1-controlled apoptopodia formation. On the one hand, EVs and apoEVs modulate immune responses in circulatory system. On the other hand, they are attributed to injury response and regeneration of local tissues.

infused MSCs has recently been revealed as a novel mechanism of MSC communication with the recipient microenvironment to promote tissue immunoregulation and regeneration ${ }^{23,30}$ (Fig. 1). Other than apoptosis of the infused MSCs per se, it is notable that they also induce recipient T-cell apoptosis via the Fas ligand (FASL)-FAS pathway ${ }^{109}$. The apoptotic $\mathrm{T}$ cells are then phagocytosed by macrophages and induce Treg upregulation to establish an immune balance, which contribute to autoimmune suppression and amelioration of pathological symptoms in colitis and systemic sclerosis ${ }^{109}$. Given the importance of apoptosis to MSCT, further elucidation of the mechanisms by which apoptosis contributes to MSCT is an intriguing and important matter, which would open a new window for effective application of MSCT.

\section{Production and functionality of apoEVs}

ApoEVs secreted from apoptotic cells contain diverse bioactive factors which endow them with a key role in tissue homeostatic maintenance. Traditionally, the only known apoEV population was that of apoBDs (diameters range of $1000-5000 \mathrm{~nm}$ ), although it is now understood that smaller apoEVs are simultaneously released from apoptotic cells ${ }^{21}$. As far as currently known, the production and secretion mechanisms of apoEVs share similarities with EVs but are also specifically characterized by apoptosis. As for EV formation, exosomes are generated in intracellular multivesicular bodies (MVBs) containing the several future exosomes called intraluminal vesicles (ILVs) ${ }^{110}$. MVBs primarily form through the invagination of plasma membrane and endosomal membrane based on ESCRT-dependent and -independent mechanisms, which subsequently secrete exosomes via Rab11/27/35-mediated exocytosis ${ }^{110}$ (Fig. 2). ApoEVs also contain exosomes-like subpopulation that is first formed in MVBs, but a featured molecular pathway is involved in apoEV release ${ }^{111}$. Specifically, cellular sphingosine1phosphate (S1P)/S1PRs couple with $\mathrm{G}_{\beta \gamma}$ to stimulate the actin cytoskeleton during apoptosis execution, which orchestrate the progression of apoEV release ${ }^{111}$ (Fig. 2). Therefore, apoEV production are largely controlled by the apoptotic process, as further confirmed by Caspase 3 been shown as an upstream molecule for apoEV formation ${ }^{112}$. As revealed, blockade of caspase-activated pannexin 1 channels (PANX1) promotes generation of "beads-on-astring" protrusion in monocytes, the produced apoEVs of which process are termed apoptopodia ${ }^{112,113}$. Apoptopodia controlled by the characterized mechanism represents a unique and novel way of apoptotic cell disassembly ${ }^{112}$ (Fig. 2). Notably, specific progression of apoptosis based 
on different cell types as well as physiological and pathological contexts contributes to different subtypes of apoEVs that contain distinct soluble metabolites, which also gives them a variety of functional properties ${ }^{114}$. Shotgun proteomics showed that apoEVs from human biliary epithelial cells of healthy control and cirrhosis patients possess significantly different proteomes ${ }^{115}$. It has been further validated that apoEVs contain a more active $20 \mathrm{~S}$ proteasome core than that of apoBDs; this controls their immunogenic activity ${ }^{116}$. Whether the different production mechanisms of apoEVs contribute to potential functional discrepancies among apoEV subpopulations remains to be investigated.

Compared to the characteristics of exosomes or microvesicles, apoEVs have unique membrane molecular components, such as the apoptotic marker phosphatidylserine (PtdSer) and C1q, which also possess characterized miRnomes and proteomes based on specific content distribution during apoptosis ${ }^{33,34,115,116}$. The characteristics can be used as the standard for the identification, isolation, and purification of apoEVs. It has been reported that a set of convenient purification and identification procedures, such as gradient centrifugation, shotgun proteomics, and flow cytometry analysis, have been applied in experiments ${ }^{33,115}$. Considering the high output and large size of apoEVs, it is not necessary to go through tedious ultracentrifugation isolation steps, which is more convenient and rapid for apoEV-based cell-free therapeutics application ${ }^{26,30,33}$.

Despite of the heterogeneity, apoEVs are emergingly considered as physiological regulators, which not only help the apoptotic cell clearance but also contribute to immunomodulation and regeneration ${ }^{21}$. The endothelial cell-derived apoEVs which contain miR-126 induce recipient vascular cells to express and secrete the CXC chemokine CXCL12, resulting in the recruitment of progenitor cells for protection of vessels from atherosclerosis $^{25}$. Moreover, a class of enriched interleukin 1 receptor antagonist (IL-1RA)-EVs secreted from MSCs are controlled by Fas, the receptor that initiates the extrinsic apoptotic pathway upon binding with FasL, and can accelerate wound healing ${ }^{24}$. Intriguingly, further evidence in a parabiosis mouse model, which connected green fluorescent protein (GFP) mice with apoptosisdeficient Fas mutant or Caspase $3^{-1-}$ mice, revealed that apoEVs participate in circulation to regulate distant $\mathrm{MSCs}^{33}$. It has also been reported that apoEVs induce $\mathrm{CD} 4^{+}$Treg responses and suppress $\mathrm{CD} 8^{+}$cytotoxic $\mathrm{T}$-cell responses to exert antitumor immunity ${ }^{117}$. In addition, apoEVs from donor plasma with acute human immunodeficiency virus (HIV-1) infection specifically inhibit dendritic cells (DCs) via targeting CD44 $4^{118}$. Taken together, these findings suggest that apoEVs are involved in multiple physiological contexts and pathological progressions, which may further contribute to regenerative and immunoregulatory therapeutic applications.

\section{Apoptotic cell and apoEV contributions to the therapeutic effects of MSCs}

Since it was first noted that infused MSCs undergoing extensive apoptosis, apoptotic MSCs have been revealed as effective candidates for promoting immunoregulation and tissue regeneration in various diseases ${ }^{28,29,33}$ (Table 1 and Fig. 3). Adiministration of apoptotic adipose-derived MSCs (ADMSCs) has been demonstrated to significantly improve the survival rate of rats with sepsis syndrome relative to administration of healthy ADMSCs, further attenuating damage to multiple organs and reducing circulating TNF- $\alpha$ levels as well as those of oxidative and apoptotic biomarkers $^{31}$ (Table 1 and Fig. 3). A further study has reported that apoptotic ADMSC infusion aids in the recovery from acute kidney injury (AKI), and tracing of apoptotic ADMSCs revealed engraftment in renal parenchyma $^{32}$ (Table 1 and Fig. 3). Concerning their immunomodulatory capacity, transplanted apoptotic DPSCs significantly inhibit allergic lung airway inflammation in mouse GvHD ${ }^{23}$ (Table 1 and Fig. 3). Moreover, apoptotic human BMMSCs have also been traced to the lungs of GvHD mice, where they are engulfed by phagocytes to induce IDO production, resulting in the reduction of GvHD effector cell infiltration ${ }^{29}$ (Table 1 and Fig. $3)$.

The potiential capacity of MSC-apoEVs to mediate tissue regeneration and immunomodulation in vivo has also been proposed. Under the apoptotic stimulus of TNF- $\alpha$, transplanted MSC-derived EVs have been demonstrated to promote gingival wound healing ${ }^{24}$ (Table 1 and Fig. 3). A recent study has also validated that MSC-derived apoEV infusion promotes cutaneous wound healing through polarizing surrounding macrophages to facilitate migration and proliferation of fibroblasts ${ }^{26}$ (Table 1 and Fig. 3). It is notable that delivery of apoEVs produced by allogeneic MSCs remarkably rescues the osteopenic phenotype in the apoptosis-deficient Fas mutant and Caspase $3^{-1-}$ mouse models ${ }^{33}$. Infusion of exogenous MSC-apoEVs is also effective in ameliorating osteoporosis in estrogen-deficient ovariectomized (OVX) mice $^{33}$. Mechanistic investigations showed that infused apoEVs are engulfed by recipient-impaired MSCs in vivo, with effects mediated by concerted transfer of miR-328-3p and ubiquitin ligase RNF146 to activate canonical WNT signaling for endogenous MSC recovery ${ }^{33}$ (Table 1 and Fig. 3). In heart injury, transplanted MSC-apoEVs regulate autophagy in cardiac endothelial cells, which enhance angiogenesis and improve cardiac functional recovery in a myocardial infarction model ${ }^{30}$. Mechanistically, infused MSC-apoEVs have been revealed to facilitate the translocation of transcription factor EB (TFEB) from lysosomes 


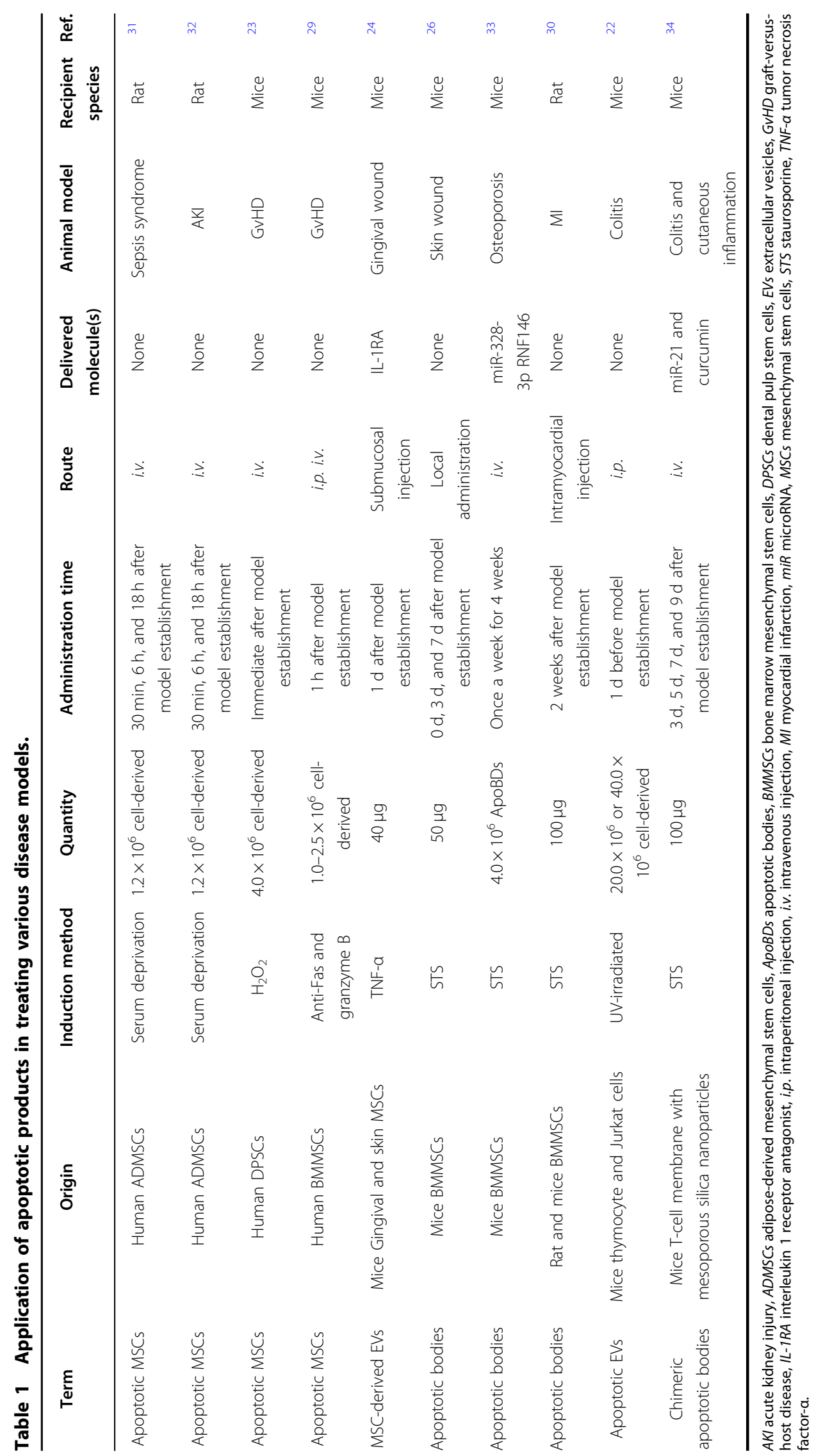




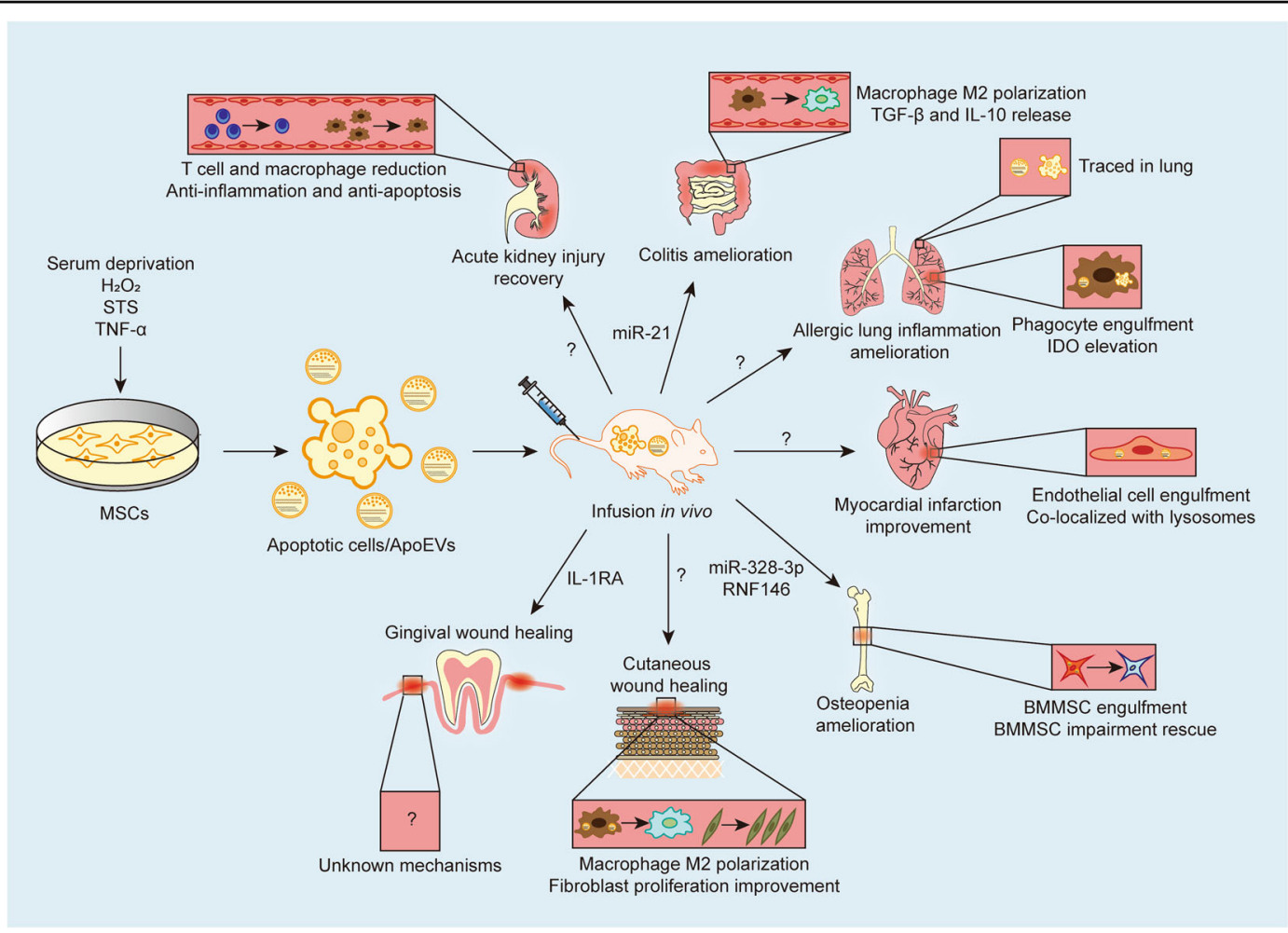

Fig. 3 Apoptosis and apoEV contribution to the therapeutic effects of MSCs. Under the multiple exogenous apoptotic stimuli, MSCs in culture can be induced to apoptosis to form apoptotic cells and apoEVs, which are collected and infused to various disease models. ApoEVs transplanted or released by infused apoptotic cells have been demonstrated to carry bioactive proteins and miRNAs to recipient cells for tissue homeostasis maintenance and immunoregulation. For examples, infused apoptotic cells and apoEVs are traced in lung and engulfed by phagocytes, which possess potent capacities of immunomodulation in inflammatory insults. ApoEVs regulate the number of immune cells and promote macrophage M2 polarization in multiple diseases including acute kidney injury, the colitis, allergic lung inflammation, and cutaneous wound healing. Apoptotic cells and apoEVs can also be engulfed by endogenous MSCs and endothelial cells, contributing to the rescue of impaired stem cells and tissue regeneration. While the therapeutic effects of apoptotic cells and apoEVs are remarkable, the mechanisms underlying molecular delivery and potential interplays between donors and recipients remain elusive.

to the nucleus, which regulates the target genes associated with autophagy and lysosomal biogenesis ${ }^{30}$ (Table 1 and Fig. 3).

As for their immunoregulatory properties, apoEVs can modulate $\mathrm{T}$-cell responses and macrophage signaling cascades in vivo, ameliorating experimental colitis ${ }^{22}$. Mechanistically, PtdSer located on the surface of apoEVs stimulates macrophages to upregulate transforming growth factor- $\beta$ (TGF- $\beta$ ) production and reduces their forkhead box O3 (FOXO3) level ${ }^{22}$ (Table 1 and Fig. 3). A recent study has also used chimeric apoEVs, which are established by loading apoEV membranes with nanoparticles and anti-inflammatory agents, to actively target macrophages to promote M2 polarization ${ }^{34}$. Animal experiments confirmed the chimeric apoEV have remarkable therapeutic effects in treating cutaneous inflammation and colitis $^{34}$. These findings collectively suggest that apoEVs are key mediators of MSCT and that apoEV administration is a promising cell-free therapeutic strategy.
As reported, the preparation processes of apoptotic MSCs did not go through isolation and purification, the therapeutic effects of apoptotic MSC transplantation may be attributed to the existence of apoEVs. The therapeutic effects of apoEVs, as well as apoptotic cells, are mainly relied on the phagocytosis of recipient cells. Although there are few reports about the characteristic comparison between apoptotic MSCs and apoEVs, it has been demonstrated that apoEVs contain a more active $20 \mathrm{~S}$ proteasome core than that of apoBDs ${ }^{116}$. Therefore, the transfer of biological signals in the form of apoEVs may be a unique and specific way.

\section{Conclusions and perspectives}

MSCT has achieved great advances in treating various diseases and realizing tissue regeneration and immunomodulation $^{8,9}$, although the challenge of how to precisely control therapeutic effects of MSCs remains to be addressed. As recent studies have reshaped our perception about apoptosis and revealed it to be critically 
involved in multiple physiological and pathological contexts, infusion of MSC-derived apoEVs has demonstrated remarkable therapeutic effects and emerged as a novel and potential cell-free therapeutic ${ }^{22,26,30,33}$. Considering the significant therapeutic effects of apoEV transplantation and the phenomenon of autonomously tissue regeneration caused by endogenous apoptotic stimuli, it is proposed that MSCs could contribute to systemic and local tissue homeostasis maintenance through autonomous apoEV production ${ }^{100-106}$. Intriguingly, accumulating studies have recently revealed that autophagic inducement enhances MSC properties in vitro, particularly in differentiation potential and its immunoregulation capacity $^{119-121}$. It has also been demonstrated that the MSC-derived inflammasomes managed by pyroptosis promote inflammatory response in vivo ${ }^{122}$. As known, immunogenic intracellular materials are released from cells under necroptosis execution, which activate immune systems and trigger extensive inflammatory response in organisms ${ }^{123}$. Accordingly, transplanted MSCs might undergo death under diverse physiological and pathological contexts, such as autophagic cell death, the necroptosis or the pyroptosis for the contribution of tissue homeostasis maintenance. As the results of parabiosis mouse model shown, apoEVs participate in the circulation $^{33}$. Questions of whether EVs produced by multiple cell death processes have differential regulatory and therapeutic effects in circulation are also interesting but still unsolved in this field.

Many studies have shown the chemotaxis of infused MSCs toward injured or inflammation sites, the issue of whether apoEVs possess specific tissue chemotaxis remains elusive. As known, infused MSCs exert immense therapeutic effects through direct effects of engraftment and differentiation, as well as indirect effects of paracrine mechanism and apoptosis execution, while transplanted apoEVs carry characterized miRnomes and proteomes to exert therapeutic effects ${ }^{115,116}$. It is notable that the generation of apoEVs, particularly regarding whether apoptosis execution of transplanted MSCs occurs before or after engraftment and migration, remains elusive. Albeit not fully understood, the targeting of apoEVs is closely related to unique membrane components, such as PtdSer and $\mathrm{C}_{1} \mathrm{q}^{33,34}$, which is an important matter and worthy of investigation in this field. Extensive experiments should be performed to investigate specific targeting of infused apoEVs and the underlying mechanisms.

Compared to MSCT, apoEV therapy possesses advantages including low immunogenicity, easy storage of reagents, reduced coagulation risk, and amenability to engineering for drug delivery. Key theranostic issues, such as heterogeneity, storage condition, quality control as well as standardization of apoEVs in induction and purification, still remain to be investigated. The realization of this paradigm shift from living MSCs to apoptotic materials will surely provide innovative and promising guidance for de novo organ regeneration and immunoregulation in future translational medicine.

\section{Author details}

${ }^{1}$ Fujian Key Laboratory of Developmental and Neural Biology \& Southern Center for Biomedical Research, College of Life Sciences, Fujian Normal University, Fuzhou, Fujian 350117, China. ${ }^{2}$ South China Center of Craniofacial Stem Cell Research, Guanghua School and Hospital of Stomatology, Sun Yatsen University, Guangzhou, Guangdong 510055, China. ${ }^{3}$ Research and Development Center for Tissue Engineering, The Fourth Military Medical University, Xi'an, Shaanxi 710032, China

\section{Author contributions}

Y.F. and B.S. contributed equally to the manuscript draft and revision. L.X., X.Y., and D.W. contributed to figure as well as table design and draft. X.H. and S.S. conceived and supervised the manuscript. All authors have reviewed, revised, and approved the final version of the manuscript.

\section{Ethics statement}

No ethics approvals were required for this paper.

\section{Funding}

This work was supported by grants from the National Natural Science Foundation of China $(81771034,81570036,32000974)$, the Science Foundation of the Fujian Province (2020J01180), the Guangdong Financial Fund for HighCaliber Hospital Construction (174-2018-XMZC-0001-03-0125, D-07), the Pearl River Talent Recruitment Program (2019ZT08Y485), the National Science and Technology Major Project of the Ministry of Science and Technology of China (2018ZX10302207-001-002), the Postdoctoral Innovative Talents Support Program of China (BX20190380), and the General Program of China Postdoctoral Science Foundation (2019M663986).

\section{Conflict of interest}

The authors declare no competing interests.

\section{Publisher's note}

Springer Nature remains neutral with regard to jurisdictional claims in published maps and institutional affiliations.

Received: 19 February 2021 Revised: 25 May 2021 Accepted: 26 May 2021 Published online: 09 June 2021

\section{References}

1. McCulloch, E. A. \& Till, J. E. The radiation sensitivity of normal mouse bone marrow cells, determined by quantitative marrow transplantation into irradiated mice. Radiat. Res. 13, 115-125 (1960).

2. Friedenstein, A. J., Chailakhjan, R. K. \& Lalykina, K. S. The development of fibroblast colonies in monolayer cultures of guinea-pig bone marrow and spleen cells. Cell Tissue Kinet. 3, 393-403 (1970).

3. Friedenstein, A. J., Chailakhyan, R. K., Latsinik, N. V., Panasyuk, A. F. \& KeilissBorok, I. V. Stromal cells responsible for transferring the microenvironment of the hemopoietic tissues. Cloning in vitro and retransplantation in vivo. Transplantation 17, 331-340 (1974).

4. Horwitz, E. M. et al. Isolated allogeneic bone marrow-derived mesenchymal cells engraft and stimulate growth in children with osteogenesis imperfecta: Implications for cell therapy of bone. Proc. Natl Acad. Sci. USA 99, 8932-8937 (2002).

5. Le Blanc, K. et al. Mesenchymal stem cells for treatment of steroid-resistant, severe, acute graft-versus-host disease: a phase II study. Lancet 371, 1579-1586 (2008).

6. Lee, R. H. et al. Intravenous hMSCs improve myocardial infarction in mice because cells embolized in lung are activated to secrete the antiinflammatory protein TSG-6. Cell Stem Cell 5, 54-63 (2009).

7. Luger, D. et al. Intravenously delivered mesenchymal stem cells: systemic anti-inflammatory effects improve left ventricular dysfunction in acute 
myocardial infarction and ischemic cardiomyopathy. Circ. Res. 120, 1598-1613 (2017).

8. Carr, M. J. et al. Mesenchymal precursor cells in adult nerves contribute to mammalian tissue repair and regeneration. Cell Stem Cell 24, 240-56 e9 (2019).

9. Gao, F. et al. Mesenchymal stem cells and immunomodulation: current status and future prospects. Cell Death Dis. 7, e2062 (2016).

10. Varderidou-Minasian, S. \& Lorenowicz, M. J. Mesenchymal stromal/stem cellderived extracellular vesicles in tissue repair: challenges and opportunities. Theranostics 10, 5979-5997 (2020).

11. Zaborowski, M. P., Balaj, L., Breakefield, X. O. \& Lai, C. P. Extracellular vesicles: composition, biological relevance, and methods of study. Bioscience $\mathbf{6 5}$ 783-797 (2015).

12. He, C., Zheng, S., Luo, Y. \& Wang, B. Exosome theranostics: biology and translational medicine. Theranostics 8, 237-255 (2018).

13. Zhu, L. P. et al. Hypoxia-elicited mesenchymal stem cell-derived exosomes facilitates cardiac repair through miR-125b-mediated prevention of cell death in myocardial infarction. Theranostics 8, 6163-6177 (2018).

14. Eirin, A. et al. Mesenchymal stem cell-derived extracellular vesicles attenuate kidney inflammation. Kidney Int 92, 114-124 (2017).

15. Zhu, Y. G. et al. Human mesenchymal stem cell microvesicles for treatment of Escherichia coli endotoxin-induced acute lung injury in mice. Stem Cells 32 116-125 (2014).

16. Shigemoto-Kuroda, T. et al. MSC-derived extracellular vesicles attenuate immune responses in two autoimmune murine models: type 1 diabetes and uveoretinitis. Stem Cell Rep. 8, 1214-1225 (2017).

17. Kerr, J. F., Wyllie, A. H. \& Currie, A. R. Apoptosis: a basic biological phenomenon with wide-ranging implications in tissue kinetics. Br. J. Cancer 26, 239-257 (1972).

18. Fuchs, Y. \& Steller, H. Programmed cell death in animal development and disease. Cell 147, 742-758 (2011).

19. Nagata, S., Hanayama, R. \& Kawane, K. Autoimmunity and the clearance of dead cells. Cell 140, 619-630 (2010).

20. Taylor, R. C., Cullen, S. P. \& Martin, S. J. Apoptosis: controlled demolition at the cellular level. Nat. Rev. Mol. Cell Biol. 9, 231-241 (2008).

21. Caruso, S. \& Poon, I. K. H. Apoptotic cell-derived extracellular vesicles: more than just debris. Front Immunol. 9, 1486 (2018).

22. Chen, $\mathrm{H}$. et al. Extracellular vesicles from apoptotic cells promote TGFbeta production in macrophages and suppress experimental colitis. Sci. Rep. $\mathbf{9}$ 5875 (2019).

23. Laing, A. G., Riffo-Vasquez, Y., Sharif-Paghaleh, E., Lombardi, G. \& Sharpe, P. T. Immune modulation by apoptotic dental pulp stem cells in vivo. Immunotherapy 10, 201-211 (2018).

24. Kou, X. et al. The Fas/Fap-1/Cav-1 complex regulates IL-1RA secretion in mesenchymal stem cells to accelerate wound healing. Sci. Transl. Med. 10 eaai8524 (2018).

25. Zernecke, A. et al. Delivery of microRNA-126 by apoptotic bodies induces CXCL12-dependent vascular protection. Sci. Signal 2, ra81 (2009).

26. Liu, J. et al. Apoptotic bodies derived from mesenchymal stem cells promote cutaneous wound healing via regulating the functions of macrophages. Stem Cell Res. Ther. 11, 507 (2020).

27. Liu, Y. et al. Mesenchymal stem cell-based tissue regeneration is governed by recipient T lymphocytes via IFN-gamma and TNF-alpha. Nat. Med. 17, 1594-1601 (2011).

28. Liu, S. et al. Mesenchymal stem cells prevent hypertrophic scar formation via inflammatory regulation when undergoing apoptosis. J. Invest. Dermatol. 134, 2648-2657 (2014).

29. Galleu, A. et al. Apoptosis in mesenchymal stromal cells induces in vivo recipient-mediated immunomodulation. Sci. Transl. Med. 9, eaam7828 (2017).

30. Liu, H. et al. Donor MSCs release apoptotic bodies to improve myocardial infarction via autophagy regulation in recipient cells. Autophagy $\mathbf{1 6}$ 2140-2155 (2020).

31. Chang, C. L. et al. Impact of apoptotic adipose-derived mesenchymal stem cells on attenuating organ damage and reducing mortality in rat sepsis syndrome induced by cecal puncture and ligation. J. Transl. Med. 10, 244 (2012).

32. Chen, $\mathrm{H}$. H. et al. Additional benefit of combined therapy with melatonin and apoptotic adipose-derived mesenchymal stem cell against sepsisinduced kidney injury. J. Pineal. Res. 57, 16-32 (2014).

33. Liu, D. et al. Circulating apoptotic bodies maintain mesenchymal stem cel homeostasis and ameliorate osteopenia via transferring multiple cellular factors. Cell Res. 28, 918-933 (2018).
34. Dou, G. et al. Chimeric apoptotic bodies functionalized with natural membrane and modular delivery system for inflammation modulation. Sci. Adv. $\mathbf{6}$ eaba2987 (2020).

35. Gronthos, S., Mankani, M., Brahim, J., Robey, P. G. \& Shi, S. Postnatal human dental pulp stem cells (DPSCs) in vitro and in vivo. Proc. Natl Acad. Sci. USA 97, 13625-13630 (2000).

36. Miura, M. et al. SHED: stem cells from human exfoliated deciduous teeth. Proc. Natl Acad. Sci. USA 100, 5807-5812 (2003).

37. Seo, B.-M. et al. Investigation of multipotent postnatal stem cells from human periodontal ligament. Lancet 364, 149-155 (2004).

38. Weiss, M. L. \& Troyer, D. L. Stem cells in the umbilical cord. Stem Cell Rev. 2 155-162 (2006).

39. $\mathrm{Bi}, \mathrm{Y}$. et al. Identification of tendon stem/progenitor cells and the role of the extracellular matrix in their niche. Nat. Med. 13, 1219-1227 (2007).

40. Zhang, Q. et al. Mesenchymal stem cells derived from human gingiva are capable of immunomodulatory functions and ameliorate inflammationrelated tissue destruction in experimental colitis. J. Immunol. 183, 7787-7798 (2009).

41. Zuk, P. A. et al. Multilineage cells from human adipose tissue: implications for cell-based therapies. Tissue Eng. 7, 211-228 (2001).

42. Shi, S. \& Gronthos, S. Perivascular niche of postnatal mesenchymal stem cells in human bone marrow and dental pulp. J. Bone Min. Res. 18, 696-704 (2003).

43. Crisan, M. et al. A perivascular origin for mesenchymal stem cells in multiple human organs. Cell Stem Cell 3, 301-313 (2008).

44. Feng, J., Mantesso, A., De Bari, C., Nishiyama, A. \& Sharpe, P. T. Dual origin of mesenchymal stem cells contributing to organ growth and repair. Proc. Natl Acad. Sci. USA 108, 6503-6508 (2011).

45. Achilleos, A. \& Trainor, P. A. Neural crest stem cells: discovery, properties and potential for therapy. Cell Res. 22, 288-304 (2012).

46. Kaukua, N. et al. Glial origin of mesenchymal stem cells in a tooth mode system. Nature 513, 551-554 (2014).

47. Di Nicola, M. et al. Human bone marrow stromal cells suppress T-lymphocyte proliferation induced by cellular or nonspecific mitogenic stimuli. Blood $\mathbf{9 9}$ 3838-3843 (2002).

48. Le Blanc, K. et al. Treatment of severe acute graft-versus-host disease with third party haploidentical mesenchymal stem cells. Lancet 363, 1439-1441 (2004).

49. Puissant, B. et al. Immunomodulatory effect of human adipose tissue-derived adult stem cells: comparison with bone marrow mesenchymal stem cells. Br. J. Haematol. 129, 118-129 (2005).

50. Nauta, A. J. \& Fibbe, W. E. Immunomodulatory properties of mesenchymal stromal cells. Blood 110, 3499-3506 (2007).

51. Ren, G. et al. Mesenchymal stem cell-mediated immunosuppression occurs via concerted action of chemokines and nitric oxide. Cell Stem Cell 2, 141-150 (2008).

52. Yamaza, T. et al. Immunomodulatory properties of stem cells from human exfoliated deciduous teeth. Stem Cell Res. Ther. 1, 5 (2010).

53. Boiret, $\mathrm{N}$. et al. Characterization of nonexpanded mesenchymal progenitor cells from normal adult human bone marrow. Exp. Hematol. 33, 219-225 (2005).

54. Kortesidis, A. et al. Stromal-derived factor-1 promotes the growth, survival, and development of human bone marrow stromal stem cells. Blood 105 3793-3801 (2005).

55. Aslan, $\mathrm{H}$. et al. Osteogenic differentiation of noncultured immunoisolated bone marrow-derived CD105+ cells. Stem Cells 24, 1728-1737 (2006).

56. LV, F. J., Tuan, R. S., Cheung, K. M. \& Leung, V. Y. Concise review: the surface markers and identity of human mesenchymal stem cells. Stem Cells 32, 1408-1419 (2014).

57. Zhao, H. et al. Secretion of shh by a neurovascular bundle niche supports mesenchymal stem cell homeostasis in the adult mouse incisor. Cell Stem Cell 14, 160-173 (2014).

58. Vidovic, I. et al. alphaSMA-expressing perivascular cells represent dental pulp progenitors in vivo. J. Dent. Res. 96, 323-330 (2017).

59. Lendahl, U., Zimmerman, L. B. \& McKay, R. D. CNS stem cells express a new class of intermediate filament protein. Cell 60, 585-595 (1990).

60. Zhou, B. O., Yue, R., Murphy, M. M., Peyer, J. G. \& Morrison, S. J. Leptinreceptor-expressing mesenchymal stromal cells represent the main source of bone formed by adult bone marrow. Cell Stem Cell 15, 154-168 (2014).

61. Liu, Y. et al. PD-1 is required to maintain stem cell properties in human dental pulp stem cells. Cell Death Differ. 25, 1350-1360 (2018). 
62. Galipeau, J. \& Sensebe, L. Mesenchymal stromal cells: clinical challenges and therapeutic opportunities. Cell Stem Cell 22, 824-833 (2018).

63. Chen, C. et al. Efficacy of umbilical cord-derived mesenchymal stem cellbased therapy for osteonecrosis of the femoral head: a three-year follow-up study. Mol. Med. Rep. 14, 4209-4215 (2016).

64. Matas, J. et al. Umbilical cord-derived mesenchymal stromal cells (MSCs) for knee osteoarthritis: repeated MSC dosing is superior to a single MSC dose and to hyaluronic acid in a controlled randomized phase $1 / 1 \mathrm{I}$ trial. Stem Cells Transl. Med. 8, 215-224 (2019).

65. Xuan, K. et al. Deciduous autologous tooth stem cells regenerate dental pulp after implantation into injured teeth. Sci. Transl. Med. 10, eaaf3227 (2018).

66. Kebriaei, $P$. et al. A phase 3 randomized study of remestemcel-L versus placebo added to second-line therapy in patients with steroid-refractory acute graft-versus-host disease. Biol. Blood Marrow Transpl. 26, 835-844 (2020).

67. Yuan, X. et al. Mesenchymal stem cell therapy induces FLT3L and CD1C(+) dendritic cells in systemic lupus erythematosus patients. Nat. Commun. 10 2498 (2019).

68. Riordan, N. H. et al. Clinical feasibility of umbilical cord tissue-derived mesenchymal stem cells in the treatment of multiple sclerosis. J. Transl. Med. 16, 57 (2018).

69. Williams, A. R. \& Hare, J. M. Mesenchymal stem cells: biology, pathophysiology, translational findings, and therapeutic implications for cardiac disease. Circ. Res. 109, 923-940 (2011).

70. Kfoury, Y. \& Scadden, D. T. Mesenchymal cell contributions to the stem cell niche. Cell Stem Cell 16, 239-253 (2015).

71. Kim, S. G. et al. Dentin and dental pulp regeneration by the patient's endogenous cells. Endod. Top. 28, 106-117 (2013).

72. Gnecchi, M. et al. Paracrine action accounts for marked protection of ischemic heart by Akt-modified mesenchymal stem cells. Nat. Med. 11 367-368 (2005).

73. Gnecchi, M. et al. Evidence supporting paracrine hypothesis for Akt-modified mesenchymal stem cell-mediated cardiac protection and functional improvement. FASEB J. 20, 661-669 (2006).

74. Kinnaird, T. et al. Local delivery of marrow-derived stromal cells augments collateral perfusion through paracrine mechanisms. Circulation 109 1543-1549 (2004).

75. Choi, H., Lee, R. H., Bazhanov, N., Oh, J. Y. \& Prockop, D. J. Anti-inflammatory protein TSG-6 secreted by activated MSCs attenuates zymosan-induced mouse peritonitis by decreasing TLR2/NF-kappaB signaling in resident macrophages. Blood 118, 330-338 (2011).

76. Ling, W. et al. Mesenchymal stem cells use IDO to regulate immunity in tumor microenvironment. Cancer Res. 74, 1576-1587 (2014).

77. Wang, G. et al. Kynurenic acid, an IDO metabolite, controls TSG-6-mediated immunosuppression of human mesenchymal stem cells. Cell Death Differ. $\mathbf{2 5}$ 1209-1223 (2018).

78. Aggarwal, S. \& Pittenger, M. F. Human mesenchymal stem cells modulate allogeneic immune cell responses. Blood 105, 1815-1822 (2005).

79. Du, L. et al. IGF-2 preprograms maturing macrophages to acquire oxidative phosphorylation-dependent anti-inflammatory properties. Cell Metab. 29, 1363-75 e8 (2019)

80. Liu, S. et al. MSC transplantation improves osteopenia via epigenetic regulation of notch signaling in lupus. Cell Metab. 22, 606-618 (2015).

81. Chen, C. et al. Mesenchymal stem cell transplantation in tight-skin mice identifies miR-151-5p as a therapeutic target for systemic sclerosis. Cell Res. 27, 559-577 (2017)

82. Yang, R. et al. IFN-gamma promoted exosomes from mesenchymal stem cells to attenuate colitis via miR-125a and miR-125b. Cell Death Dis. 11, 603 (2020).

83. Mayourian, J. et al. Exosomal microRNA-21-5p mediates mesenchymal stem cell paracrine effects on human cardiac tissue contractility. Circ. Res. 122 933-944 (2018).

84. Phinney, D. G. et al. Mesenchymal stem cells use extracellular vesicles to outsource mitophagy and shuttle microRNAs. Nat. Commun. 6, 8472 (2015)

85. Fuchs, Y. \& Steller, H. Live to die another way: modes of programmed cell death and the signals emanating from dying cells. Nat. Rev. Mol. Cell Biol. 16, 329-344 (2015).

86. Doerflinger, M. et al. Flexible usage and interconnectivity of diverse cell death pathways protect against intracellular infection. Immunity 53,533-47 e7 (2020).
87. Zhang, Y., Chen, X., Gueydan, C. \& Han, J. Plasma membrane changes during programmed cell deaths. Cell Res 28, 9-21 (2018).

88. Coleman, M. L. et al. Membrane blebbing during apoptosis results from caspase-mediated activation of ROCK I. Nat. Cell Biol. 3. 339-345 (2001).

89. Sisirak, V. et al. Digestion of chromatin in apoptotic cell microparticles prevents autoimmunity. Cell 166, 88-101 (2016).

90. Cabral-Piccin, M. P. et al. Apoptotic CD8 T-lymphocytes disable macrophagemediated immunity to Trypanosoma cruzi infection. Cell Death Dis. 7, e2232 (2016)

91. Taylor, J. J., Pape, K. A., Steach, H. R. \& Jenkins, M. K. Humoral immunity. Apoptosis and antigen affinity limit effector cell differentiation of a single naive B cell. Science 347, 784-787 (2015).

92. Morris, A. B. et al. Signaling through the inhibitory Fc receptor FcgammaRIIB induces CD8(+) $\mathrm{T}$ cell apoptosis to limit $\mathrm{T}$ cell immunity. Immunity $\mathbf{5 2}$ 136-50 e6 (2020).

93. Rodriguez-Manzanet, R. et al. T and B cell hyperactivity and autoimmunity associated with niche-specific defects in apoptotic body clearance in TIM-4deficient mice. Proc. Natl Acad. Sci. USA 107, 8706-8711 (2010).

94. Herrmann, M. et al. Impaired phagocytosis of apoptotic cell material by monocyte-derived macrophages from patients with systemic lupus erythematosus. Arthritis Rheum. 41, 1241-1250 (1998).

95. Kawane, K. et al. Requirement of DNase II for definitive erythropoiesis in the mouse fetal liver. Science 292, 1546-1549 (2001).

96. Kawane, K. et al. Chronic polyarthritis caused by mammalian DNA that escapes from degradation in macrophages. Nature 443, 998-1002 (2006)

97. Berda-Haddad, $Y$, et al. Sterile inflammation of endothelial cell-derived apoptotic bodies is mediated by interleukin-1alpha. Proc. Natl Acad. Sci. USA 108, 20684-20689 (2011)

98. Wickman, G. R. et al. Blebs produced by actin-myosin contraction during apoptosis release damage-associated molecular pattern proteins before secondary necrosis occurs. Cell Death Differ. 20, 1293-1305 (2013).

99. Fogarty, C. E. \& Bergmann, A. Killers creating new life: caspases drive apoptosis-induced proliferation in tissue repair and disease. Cell Death Differ 24, 1390-1400 (2017).

100. Chera, S. et al. Apoptotic cells provide an unexpected source of Wnt3 signaling to drive hydra head regeneration. Dev. Cell 17, 279-289 (2009)

101. Gupta, K. H. et al. Apoptosis and compensatory proliferation signaling are coupled by Crkl-containing microvesicles. Dev. Cell 41, 674-84 e5 (2017).

102. Brock, C. K. et al. Stem cell proliferation is induced by apoptotic bodies from dying cells during epithelial tissue maintenance. Nat. Commun. 10, 1044 (2019)

103. Koren, E. et al. ARTS mediates apoptosis and regeneration of the intestinal stem cell niche. Nat. Commun. 9, 4582 (2018).

104. limuro, Y. et al. NFkappaB prevents apoptosis and liver dysfunction during liver regeneration. J. Clin. Invest 101, 802-811 (1998).

105. Malato, Y. et al. NF-kappaB essential modifier is required for hepatocyte proliferation and the oval cell reaction after partial hepatectomy in mice. Gastroenterology 143, 1597-608 e11 (2012).

106. Neumann, B. et al. EFF-1-mediated regenerative axonal fusion requires components of the apoptotic pathway. Nature 517, 219-222 (2015).

107. Perez-Garijo, A., Fuchs, Y. \& Steller, H. Apoptotic cells can induce nonautonomous apoptosis through the TNF pathway. Elife 2, e01004 (2013).

108. Li, Y. \& Lin, F. Mesenchymal stem cells are injured by complement after their contact with serum. Blood 120, 3436-3443 (2012).

109. Akiyama, K. et al. Mesenchymal-stem-cell-induced immunoregulation involves FAS-ligand-/FAS-mediated T cell apoptosis. Cell Stem Cell 10, 544-555 (2012).

110. Catalano, M. \& O'Driscoll, L. Inhibiting extracellular vesicles formation and release: a review of EV inhibitors. J. Extracell. Vesicles 91703244 (2020).

111. Park, S. J. et al. Molecular mechanisms of biogenesis of apoptotic exosome-like vesicles and their roles as damage-associated molecular patterns. Proc. Natl Acad. Sci. USA 115, E11721-E11730 (2018)

112. Atkin-Smith, G. K. et al. A novel mechanism of generating extracellular vesicles during apoptosis via a beads-on-a-string membrane structure. Nat Commun. 6, 7439 (2015).

113. Poon, I. K. et al. Unexpected link between an antibiotic, pannexin channels and apoptosis. Nature 507, 329-334 (2014).

114. Poon, I. K. H. et al. Moving beyond size and phosphatidylserine exposure: evidence for a diversity of apoptotic cell-derived extracellular vesicles in vitro. J. Extracell. Vesicles 8, 1608786 (2019). 
115. Lleo, A. et al. Shotgun proteomics: identification of unique protein profiles of apoptotic bodies from biliary epithelial cells. Hepatology 60, 1314-1323 (2014).

116. Dieude, M. et al. The $20 \mathrm{~S}$ proteasome core, active within apoptotic exosomelike vesicles, induces autoantibody production and accelerates rejection. Sci. Transl. Med. 7, 318ra200 (2015).

117. Xie, Y. et al. Tumor apoptotic bodies inhibit CTL responses and antitumor immunity via membrane-bound transforming growth factor-beta1 inducing CD8+ T-cell anergy and CD4+ Tr1 cell responses. Cancer Res. 69, 7756-7766 (2009).

118. Frleta, D. et al. HIV-1 infection-induced apoptotic microparticles inhibit human DCs via CD44. J. Clin. Invest 122, 4685-4697 (2012).
119. Ma, Y. et al. Autophagy controls mesenchymal stem cell properties and senescence during bone aging. Aging Cell 17, e12709 (2018).

120. Cen, S. et al. Autophagy enhances mesenchymal stem cell-mediated CD4(+) T cell migration and differentiation through CXCL8 and TGF-beta1. Stem Cell Res. Ther. 10, 265 (2019).

121. Regmi, S. et al. Enhanced viability and function of mesenchymal stromal cell spheroids is mediated via autophagy induction. Autophagy 2020: 1-20.

122. Chen, Y. et al. Mesenchymal stromal cells directly promote inflammation by canonical NLRP3 and non-canonical caspase-11 inflammasomes. EBioMedicine 32, 31-42 (2018)

123. Pasparakis, M. \& Vandenabeele, P. Necroptosis and its role in inflammation. Nature 517, 311-320 (2015) 